\title{
Utilidad de la elastografía cuantitativa en el diagnóstico de las lesiones sólidas del páncreas
}

\section{Usefulness of quantitative elastography in the diagnosis of solid lesions of the páncreas}

Claudia Patricia Díaz-Tobar. ${ }^{1}$

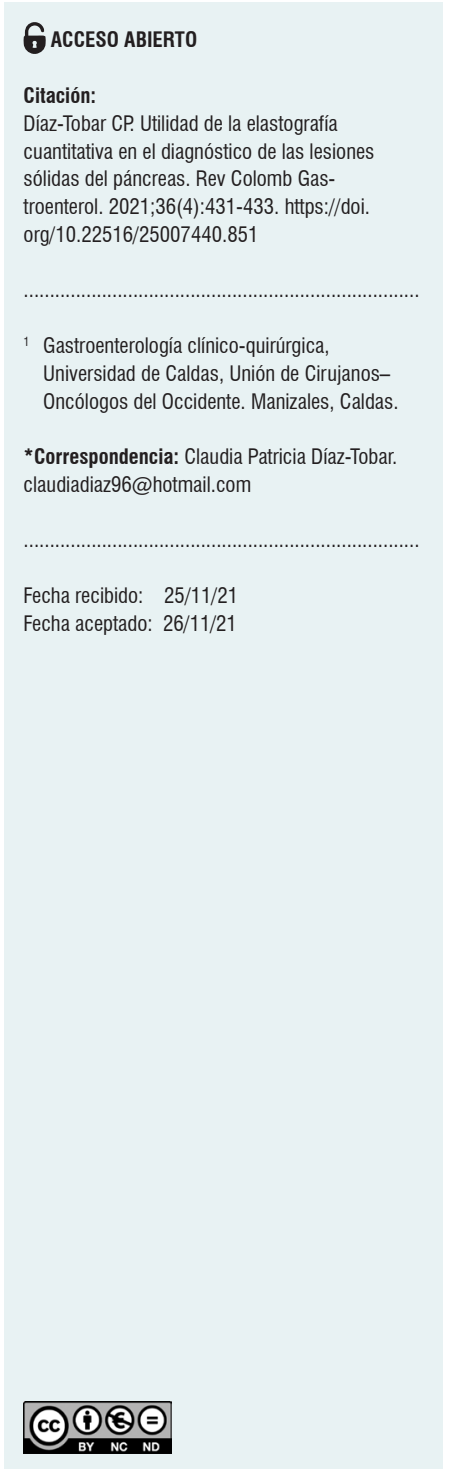

Las lesiones sólidas del páncreas en general cuentan con tasas de supervivencia inferiores al $5 \%$ a los 5 años, a pesar de los avances en los métodos diagnósticos ${ }^{(1)}$.

Con la llegada de la ultrasonografía endoscópica (USE) asociada con la biopsia por aspiración con aguja fina (FNA), el diagnóstico de las lesiones pancreáticas ha mejorado notablemente, con una sensibilidad y especificidad reportadas entre el $85 \%$ y $100 \%$, respectivamente; pero es una técnica demandante, no exenta de complicaciones tales como sangrado, infección y pancreatitis aguda. Además, es relativamente frecuente que se reporten falsos negativos, hasta en un $20 \%$ a $40 \%$, en pacientes con lesiones sólidas del páncreas, en especial en aquellos con pancreatitis crónica, lo cual se convierte en un desafío diagnóstico ${ }^{(2,3)}$.

Dadas las limitaciones presentadas por la USE con FNA, se han desarrollado nuevas tecnologías que ayudan a mejorar su rendimiento diagnóstico, tales como los medios de contraste, endomicroscopia confocal y la elastografía.

La elastografía es una técnica que evalúa la dureza de los tejidos por medio de su elasticidad, semejante a una palpación virtual ${ }^{(4)}$. El primero en evaluar la elastografía en páncreas fue Giovannini en 2006 en un estudio con 49 pacientes, en el que describió una escala de 1 a 5 para definir diferentes patrones desde el tejido normal (puntaje 1) hasta el adenocarcinoma (puntaje 5), con una sensibilidad del $100 \%$, pero una especificidad del $67 \%$, y la describe como una nueva aplicación de la endosonografía para diferenciar el tejido benigno de las lesiones malignas ${ }^{(5)}$. Iglesias y colaboradores realizaron un estudio con 130 pacientes, en el que reportaron una escala de 1 a 4 para diferenciar un páncreas normal de un tumor en páncreas ${ }^{(2)}$, similar a lo reportado por Giovannini.

La elastografía tiene 2 patrones de evaluación: uno cualitativo y otro cuantitativo. La elastografía cualitativa evalúa la dureza de los tejidos mediante la interpretación de un mapa de colores: el verde representa la rigidez media, el rojo, la más blanda, y el azul, la más rígida ${ }^{(6)}$, lo que demuestra una alta sensibilidad en la valoración de las lesiones sólidas del páncreas de un $95 \%$ a $98 \%$, pero con una baja sensibilidad del $42 \%$ al $76 \%$, que la convierte en una herramienta útil para el diagnóstico ${ }^{(7)}$. La elastografía cuantitativa mide la dureza del tejido objetivo comparándola con un área de referencia alrededor de la lesión, lo que permite calcular el strain ratio (SR) y el histograma; de este modo se determina la naturaliza benigna o maligna de las lesiones ${ }^{(6)}$.

El histograma evalúa la dureza del tejido mediante la evaluación de un área seleccionada que contenga al menos el $50 \%$ de la lesión, sin incluir un tejido de referencia; el software convierte la imagen seleccionada en una escala de colores que determina la dureza del 
tejido (0 [duro] a 255 [blando] $)^{(7)}$. Se han reportado diferentes puntos de corte en el histograma para el diagnóstico diferencial de las lesiones de páncreas, entre ellos, valores mayores de 80 como benignas y menores de 80 como malignas ${ }^{(7)}$. Iglesias y colaboradores reportaron un punto de corte de menor de 50 para malignidad, con una precisión superior al $98 \%{ }^{(8)}$. Popescu y colaboradores reportaron una sensibilidad para el histograma del $93,4 \%$ a $91,4 \%$, pero con una especificidad variable del $66 \%$ al $87,9 \%$ con un punto de corte de $175^{(6-9)}$.

El SR mide el área objetivo comparado con un área de referencia usando un tejido alrededor del páncreas ${ }^{(6)}$. Los estudios reportaron diferentes puntos de corte en el SR para clasificar las lesiones pancreáticas. En 2011, Itokawa y colaboradores realizaron un estudio con 109 pacientes y reportaron un SR de 23,6 para masas pancreáticas no malignas y de 39,08 para cáncer de páncreas, con una sensibilidad del $85 \%$ y una especificidad del $91 \%{ }^{(10)}$. En 2017, Kim y colaboradores reportaron un SR de 3,78 para páncreas normal, 8,2 para pancreatitis crónica y 21,8 para cáncer de páncreas, con una sensibilidad del $95 \%$ y una especificidad del $96 \%$ para el diagnóstico de malignidad ${ }^{(11)}$. Iglesias y colaboradores reportaron un punto de corte para el SR mayor de 10 para determinar las lesiones pancreáticas como malignas con una precisión diagnóstica del $98 \%{ }^{(8)}$. Dawwas y colaboradores reportaron una sensibilidad del $100 \%$ con una pobre especificidad del $16 \%{ }^{(9)}$. Se han realizado varios metaanálisis para determinar la utilidad de la elastografía en el diagnóstico de las lesiones pancreáticas y se reportó una alta sensibilidad entre el $95 \%$ y el $99 \%$, con una especificidad variable del $67 \%$ al $76 \%{ }^{(9)}$.

Esta variabilidad en el punto de corte se ha relacionado con la falta de estandarización en la localización del punto de referencia ${ }^{(6-9)}$. Otras limitaciones reportadas en la realización de la elastografía son los artefactos, reverberación, ser dependiente del operador, variabilidad interobservador y la falta de uniformidad de los sistemas comerciales ${ }^{(12)}$.

En el estudio de corte transversal prospectivo, realizado por los doctores Martín Gómez Zuleta, Oscar Ruiz y Diego
Cano, publicado en este número, se reportaron 71 pacientes, se tomó como punto de corte 22 puntos para el SR en las lesiones malignas de páncreas, y se encontró una sensibilidad del $94 \%$ con una especificidad del $89,3 \%$ en la detección de las lesiones sólidas malignas del páncreas. Se concluyó que la utilidad actual de la elastografía cuantitativa en las lesiones sólidas del páncreas es mejorar la precisión de la FNA al seleccionar de una manera más objetiva el área más sospechosa para realizar la punción.

La elastografía en el diagnóstico de lesiones sólidas del páncreas tiene una gran utilidad, al permitir orientar sobre la naturaleza de las lesiones y realizar una FNA dirigida a la zona de mayor sospecha diagnóstica, con unas altas tasas de sensibilidad reportadas. Se encontraron varias limitaciones de la elastografía como ser dependiente del operador, falta de estandarización de la técnica, variabilidad interobservador, diferentes puntos de corte reportados en la literatura tanto para el histograma como para el SR y las diferentes tasas en la especificidad reportadas en la literatura.

Las limitaciones del estudio realizado por Gómez y colaboradores son las reportadas en la literatura en general, mencionadas en el párrafo anterior, determinadas principalmente por la falta en la estandarización de la técnica y la falta de uniformidad en el punto de corte del SR. Otras limitaciones encontradas fueron los procedimientos realizados solo por un endoscopista y en un solo centro hospitalario, no se reporta si la valoración de las muestras fueron realizadas por un patólogo experto en patología pancreática y en lectura de este tipo de muestras. Como ventajas, hay un estudio con datos de nuestra población, con una cantidad adecuada de pacientes, en el cual se utiliza un método cuantitativo como el SR, lo cual proporciona una evaluación de las lesiones que sea menos dependiente del operador.

Tener mejores herramientas en el diagnóstico de lesiones pancreáticas permitirá un diagnóstico oportuno, evitará la realización de punciones no exentas de complicaciones (lo cual se verá reflejado también en el costo médico) y disminuirá la angustia del paciente al no tener un diagnóstico de su enfermedad que permita iniciar un tratamiento a tiempo.

\section{REFERENCIAS}

1. Okasha HH, Mahdy RE, Elkholy S, Hassan MS, El-Mazny AN, Hadad KEE, Saeed M, El-Nady M, Elbalky OS, Ashraf A, El-Magd AA, Awad A. Endoscopic ultrasound (EUS) elastography and strain ratio, could it help in differentiating malignant from benign pancreatic lesions? Medicine (Baltimore). 2018;97(36):e11689. https://doi.org/10.1097/MD.0000000000011689
2. Iglesias-Garcia J, Lindkvist B, Lariño-Noia J, DomínguezMuñoz JE. Endoscopic ultrasound elastography. Endosc Ultrasound. 2012;1(1):8-16. https://doi.org/10.7178/eus.01.003

3. Mei M, Ni J, Liu D, Jin P, Sun L. EUS elastography for diagnosis of solid pancreatic masses: a meta-analysis. Gastrointest Endosc. 2013;77(4):578-89.

https://doi.org/10.1016/j.gie.2012.09.035 
4. Seicean A, Mosteanu O, Seicean R. Maximizing the endosonography: The role of contrast harmonics, elastography and confocal endomicroscopy. World J Gastroenterol. 2017;23(1):25-41.

https://doi.org/10.3748/wjg.v23.i1.25

5. Giovannini M, Hookey LC, Bories E, Pesenti C, Monges G, Delpero JR. Endoscopic ultrasound elastography: the first step towards virtual biopsy? Preliminary results in 49 patients. Endoscopy. 2006;38(4):344-8.

https://doi.org/10.1055/s-2006-925158

6. Chantarojanasiri T, Kongkam P. Endoscopic ultrasound elastography for solid pancreatic lesions. World J Gastrointest Endosc. 2017;9(10):506-513. https://doi.org/10.4253/wjge.v9.i10.506

7. Costache MI, Cazacu IM, Dietrich CF, Petrone MC, Arcidiacono PG, Giovannini M, Bories E, Garcia JI, Siyu S, Santo E, Popescu CF, Constantin A, Bhutani MS, Saftoiu A. Clinical impact of strain histogram EUS elastography and contrast-enhanced EUS for the differential diagnosis of focal pancreatic masses: A prospective multicentric study. Endosc Ultrasound. 2020;9(2):116-121. https://doi.org/10.4103/eus.eus_69_19
8. Iglesias-Garcia J, Lariño-Noia J, Domínguez-Muñoz JE. New diagnostic techniques for the differential diagnosis of pancreatic mass: Elastography helps me 100. Endosc Ultrasound. 2017;6(Suppl 3):S115-S118. https://doi.org/10.4103/eus.eus_76_17

9. Popescu A, Săftoiu A. Can elastography replace fine needle aspiration? Endosc Ultrasound. 2014;3(2):109-17. https://doi.org/10.4103/2303-9027.123009

10. Itokawa $F$, Itoi $T$, Sofuni $A$, Kurihara $T$, Tsuchiya $T$, Ishii K, Tsuji S, Ikeuchi N, Umeda J, Tanaka R, Yokoyama N, Moriyasu F, Kasuya K, Nagao T, Kamisawa T, Tsuchida A. EUS elastography combined with the strain ratio of tissue elasticity for diagnosis of solid pancreatic masses. J Gastroenterol. 2011;46(6):843-53. https://doi.org/10.1007/s00535-011-0399-5

11. Kim SY, Cho JH, Kim YJ, Kim EJ, Park JY, Jeon TJ, Kim YS. Diagnostic efficacy of quantitative endoscopic ultrasound elastography for differentiating pancreatic disease. J Gastroenterol Hepatol. 2017;32(5):1115-1122. https://doi.org/10.1111/jgh.13649

12. Sigrist RMS, Liau J, Kaffas AE, Chammas MC, Willmann JK. Ultrasound Elastography: Review of Techniques and Clinical Applications. Theranostics. 2017;7(5):1303-1329. https://doi.org/10.7150/thno.18650 